\title{
Effects of Different Conditions on the Removal of Dye from Reactive Dye Wastewater Using Inorganic-Organic Composite Polymer
}

\author{
Khai Ern Lee, Norhashimah Morad, Tjoon Tow Teng, and Beng Teik Poh
}

\begin{abstract}
A new composite material (MCPAM), which was prepared from magnesium chloride premixed with polyacrylamide, was applied in flocculating simulated reactive dye (Cibacron Red FN-R) wastewater. Effects of different conditions such as $\mathrm{pH}$, temperature, concentration of dye and dosage of composite polymer were taken into account to investigate their effect on dye removal (\%). pH 12 gave the maximum dye removal (\%). Flocculation of simulated reactive dye wastewater was carried out in the range of $20-50^{\circ} \mathrm{C}$ to investigate the effect of temperature on the dye removal $(\%)$. Results showed that the dye removal (\%) decreased with the increase of temperature. An interaction effect was observed between temperature and concentration of dye where the dye removal (\%) decreased with the increase of dye concentration from 200 to $500 \mathrm{mg} / \mathrm{L}$ at $20^{\circ} \mathrm{C}$. However, at temperature beyond $30^{\circ} \mathrm{C}$, an inverse behaviour was observed. Effect of dosage on the dye removal (\%) was studied with respect to concentration of dye. The dye removal $(\%)$ was found to increase to a maximum value of $99 \%$ and decrease thereafter, indicating the re-suspension of destabilized flocs into the supernatant.
\end{abstract}

Index Terms-Inorganic-organic composite polymer, flocculation, reactive dye, wastewater

\section{INTRODUCTION}

Coagulation-flocculation is an important unit operation in the industries for wastewater treatment and sludge dewatering [1-3]. Coagulation of dissolved and colloidal substances in wastewater is explained by Derjaguin - Landau - Verwey - Overbeen theory (DLVO theory) whereby coagulation is a process that overcomes the interparticle repulsive energy barrier and induces the aggregation of dissolved and colloidal substances. The introduction of inorganic substances such as aluminium and iron based coagulants helps in neutralizing the surface charge of suspended particles, facilitating particle aggregation and finally settling under gravity as a result of electrical double layer compression [4]. Flocculation process is referred to the addition of floc-forming chemical reagent to the wastewater to agglomerate non-settable and slow-settling colloidal solids Flocculation plays a major role in transporting contaminants out from aquatic environment by bridging the aggregated flocs to form larger agglomerates in the presence of

Manuscript received February 1, 2012. This work was supported in part by the Universiti Sains Malaysia in the form of postgraduate fellowship as well as Research University grant 1001/PTEKIND/814049.

Khai Ern Lee, Norhashimah Morad, Tjoon Tow Teng and Beng Teik Poh are with School of Industrial Technology, Universiti Sains Malaysia, 11800 Penang, Malaysia. (e-mail: nhashima@usm.my) polymeric materials [5-6].

Various polymeric materials have been developed in recent years for coagulation-flocculation purpose. Among them, composite materials have received increasing attention due to their better performance compared to that of conventional inorganic-based coagulants, and their lower cost compared to organic-based flocculants. There are a few terminologies used to address composite materials, such as: composite coagulant [7], composite flocculant [8], composite polymer [9], as well as hybrid polymer [2-3]. Composite materials refer to the introduction of other effective components into the matrix of original materials to enhance the aggregating power of the materials [10]. Due to the synergetic effect of two components in one material, therefore, composite materials pose a superior performance as compared to that of individual component. Generally, composite materials that are used for coagulation-flocculation purpose can be classified into a few groups: (i) inorganic-inorganic, (ii) inorganic-organic, (iii) inorganic-natural polymer, (iv) inorganic-biopolymer and (v) organic-organic composite. Inorganic-organic composite particularly have received the most attention in treating wastewater. The molecular weight and size of inorganic coagulants are much lower than that of organic flocculants, resulting in the aggregating capacity to remain lower than that of organic flocculants. Such a limitation can be resolved by introducing water soluble polymers e.g. polyacrylamide (PAM) to be composed with inorganic coagulants to prepare inorganic-organic composite polymers [2-3]. With this modification, the bridging capacity of the materials hence can be improved. The application of these inorganic-organic composite materials required only one unit operation which is the flocculation system, compared to the conventional bi-operational system: coagulation-flocculation. Such an innovation is essential in reducing the wastewater treatment processing time especially for those industries that are dealing with large volume of wastewater, particularly, textile industry.

Textile dyeing and printing process produce large quantities of wastewater which are often characterized with high temperature, $\mathrm{pH}, \mathrm{COD}$ and colour content [11]. Therefore, the effluent from the dyeing process needs to be treated before being discharged to reduce the impact to the environment. Reactive dye particularly has been extensively used in the textile industry to colour cellulosic-based fabric. To fix the colour on the cellulosic fabric, reactive dyes are applied under basic condition at elevated temperature [11]. The aim of the present work was to examine the ability of 
MCPAM composite polymer as a flocculant to treat simulated dye wastewater at different conditions considering $\mathrm{pH}$, concentration of dye, dosage as well as temperature which often act as important parameters that affect the flocculation efficiency.

\section{MATERIALS AND METHODS}

\section{A. Materials}

Acrylamide (AM) (>99\% purity, Merck) was used without further purification. Ammonium persulphate (AR, Systerm) and sodium bisulfite (GR, Acros Organics) were used as redox initiators. Magnesium chloride ( $\geq 99 \%$, Bendosen) was used as received. Deioinized water was used in the preparation of MCPAM composite polymer.

\section{B. Preparation of Inorganic-Organic Composite Polymer}

As described in our previous paper [12], inorganic-organic composite polymer (MCPAM) was prepared by premixing magnesium chloride and polyacrylamide in aqueous solution with the composition of $90 \% \mathrm{MgCl}_{2}: 10 \%$ PAM (w:w). Prior to any application, the prepared aqueous solution of MCPAM composite polymer was aged for 24 hours at room temperature. Fig. 1 shows the transmission electron microscopy (TEM) image of MCPAM composite polymer. A tree-root liked structure is observed for polyacrylamide and magnesium chloride which appears in black spots is embedded on the polyacrylamide chain.

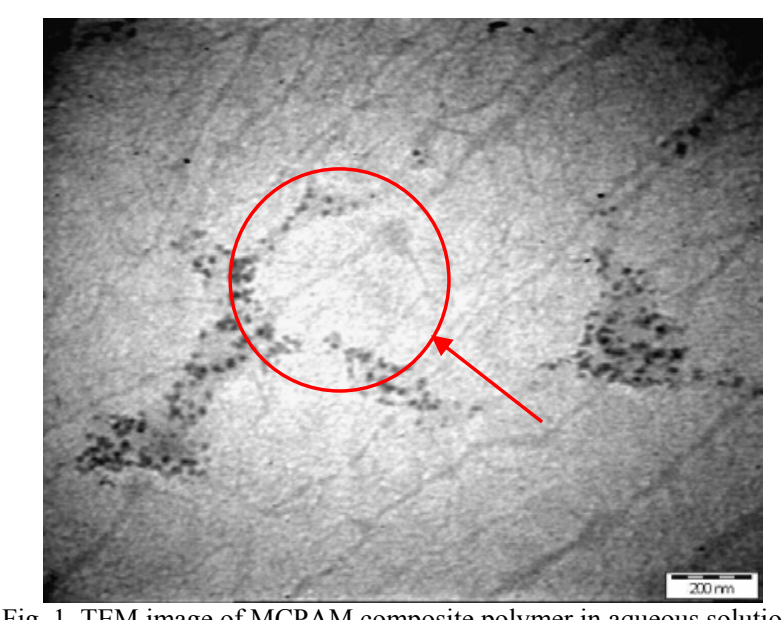

Fig. 1. TEM image of MCPAM composite polymer in aqueous solution.

\section{Flocculation Procedure}

Cibacron Red FN-R (CI Reactive Red 238, $\mathrm{C}_{29} \mathrm{H}_{15} \mathrm{O}_{13} \mathrm{~S}_{4} \mathrm{ClFN}_{7} \mathrm{Na}_{4}$, molecular weight: $944.2 \mathrm{~g} \mathrm{~mol}^{-1}$ ) was used to prepare simulated reactive dye wastewater. MCPAM composite polymer was used to flocculate different concentrations of Cibacron Red FN-R aqueous solution. $\mathrm{HCl}$ and $\mathrm{NaOH}$ were used to adjust the $\mathrm{pH}$ of the simulated reactive wastewater. A high torque stirrer (IKA LABORTECHNIK RW20DZM.n) was used in flocculating simulated reactive wastewater as shown in Fig. 2. The temperature of dye wastewater was controlled using thermostatic water bath (KBLee 2010). MCPAM composite polymer was introduced into the simulated reactive dye wastewater and an agitation speed of 100rpm was applied for 5 minutes. Flocs formed were allowed to settle for 30 minutes. The dye residual that remained in the supernatant was measured with spectrophotometer (HACH DR2800) at $\lambda_{\max }=$ $540 \mathrm{~nm}$.

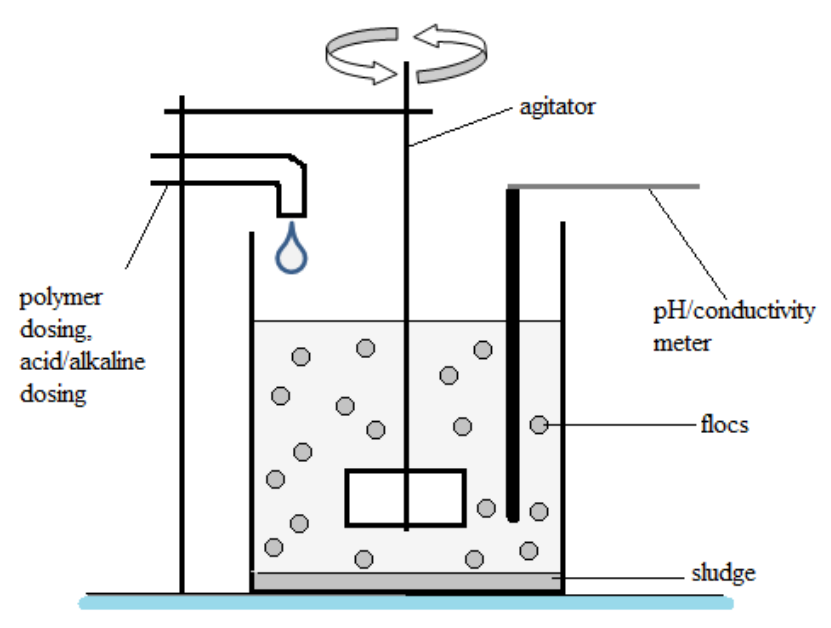

Fig. 2. Schematic diagram of flocculation of reactive dye wastewater.

\section{RESULTS AND DISCUSSION}

\section{A. Effect of $p H$}

Magnesium chloride was selected to be composed with polyacrylamide to prepare MCPAM composite polymer as magnesium chloride works well in basic region [13]. With the application of MCPAM composite polymer, no drastic $\mathrm{pH}$ adjustment is needed for the flocculation of reactive dye wastewater. It is because the working $\mathrm{pH}$ of the reactive dyeing process is usually high $(\mathrm{pH}>11)$ to fix the colour on the cellulosic fabric [8]. Therefore, the $\mathrm{pH}$ range was fixed at 11.25 to 12.50 to determine the best working $\mathrm{pH}$ in flocculating $500 \mathrm{mg} / \mathrm{L}$ reactive dye wastewater using a dosage of $1000 \mathrm{mg} / \mathrm{L}$ MCPAM composite polymer. It is observed in Fig. 3 that the dye removal (\%) increases with $\mathrm{pH}$ where it achieves the highest efficiency at $\mathrm{pH} 12$; however, it decreases thereafter. Such an observation can be attributed to almost all $\mathrm{Mg}^{2+}$ ions from MCPAM composite polymer have been converted to precipitable hydroxide by the excess $\mathrm{OH}^{-}$ ion when the $\mathrm{pH}$ increases beyond 12. MCPAM composite polymer is dissociated into $\left[\mathrm{Mg}^{2+} \cdot 2 \mathrm{Cl}^{-}\right]-\mathrm{PAM}$ when it is introduced into the reactive dye wastewater as shown in the proposed mechanism in Equation 1. Certain amount of $\mathrm{OH}-$ ions are needed to bind with $\left[\mathrm{Mg}^{2+} \cdot 2 \mathrm{Cl}^{-}\right]$-PAM to form Dye- $\mathrm{Mg}(\mathrm{OH})_{2}$-PAM (flocs) and release $\mathrm{Cl}^{-}$into the supernatant. Excess of $\mathrm{OH}^{-}$ions (beyond $\mathrm{pH}$ 12) induces the forming of $\mathrm{Mg}(\mathrm{OH})_{2}$-PAM precipitable species that without binding any dye molecule which in turn results in the decrease of dye removal (\%)

$\mathrm{Dye}+2 \mathrm{OH}^{-}+\left[\mathrm{Mg}^{2+} \cdot 2 \mathrm{Cl}^{-}\right]-\mathrm{PAM} \rightleftharpoons$ Dye- $\mathrm{Mg}(\mathrm{OH})_{2}-\mathrm{PAM}$ (flocs) $+\mathrm{Cl}^{-}$ 


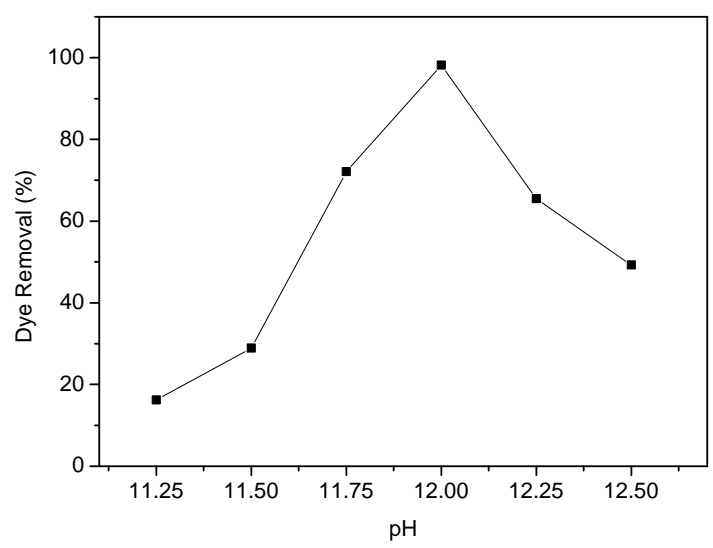

Fig. 3 Effect of $\mathrm{pH}$ on the dye removal (\%).

\section{B. Effect of Temperature}

Textile wastewater is often discharged at high temperature. Therefore, a temperature range of 20 to $50^{\circ} \mathrm{C}$ has been taken into account to investigate the effect of temperature on the flocculation of reactive dye. The experiments were carried out for different dye concentrations $(200-500 \mathrm{mg} / \mathrm{L})$ at $\mathrm{pH} 12$ using a dosage of $500 \mathrm{mg} / \mathrm{L}$ MCPAM composite polymer. The results in Fig. 4 show that an increase in the temperature limits the efficiency of flocculation. This could be due to the dye molecules with electrostatic bonding onto the MCPAM composite polymer chains are disrupted. The dye molecule has been released into the supernatant at elevated temperature which somewhat it reduces the dye removal (\%) [14]. The dye removal (\%) decreases from 87 to $61 \%$ with the increase of dye concentration from 200 to $500 \mathrm{mg} / \mathrm{L}$ at $20^{\circ} \mathrm{C}$. However, an inverse behaviour is observed at temperature beyond $30^{\circ} \mathrm{C}$. An unparallel effect is observed between dye concentration and temperature. This implies that there is an interaction effect between these two factors. The results indicate that the suitable temperature to flocculate reactive dye wastewater is $30^{\circ} \mathrm{C}$ where it is near to the room temperature. Thus, it is recommended that the reactive dye wastewater need to be cool down to room temperature prior to any flocculation process take place.

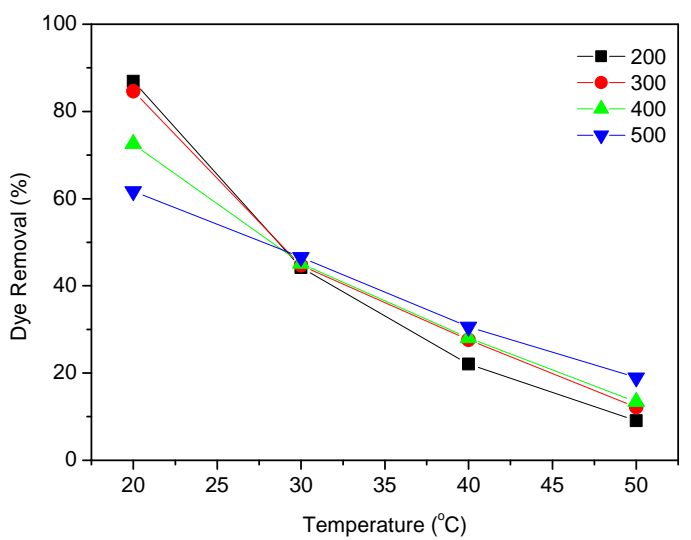

Fig. 4. Effects of temperature and initial dye concentration on the dye removal.

\section{Effect of Dosage}

The effect of dosage on the dye removal (\%) was studied with respect to concentration of dye. The effect of dosage on the dye removal (\%) is shown in Fig. 5. A maximum dye removal (\%) of $99 \%$ is achieved for all dye concentrations $(200-500 \mathrm{mg} / \mathrm{L})$. Overall, a similar trend is observed for all dye concentrations where the dye removal (\%) increases to a maximum value and decrease thereafter. Such an observation is attributed to the reactive dye needs certain dosage of MCPAM composite polymer in flocculation process to destabilize the molecule of reactive dye; further addition of dosage causes the re-suspension of destabilized flocs into the supernatant. A ratio of MCPAM composite polymer dosage/dye concentration at the maximum dye removal (\%) is used to estimate the number of composite polymer needed to flocculate one dye molecule. It is noted that the concentration of dye decreases from 500 to $200 \mathrm{mg} / \mathrm{L}$, the ratio of dye concentration/dosage of MCPAM composite polymer increase from 3 to 6 . This implies that the probability for dye molecule and MCPAM composite polymer to contact and capture each other to form flocs is getting lower with the decrease of concentration of dye. Therefore, a higher ratio of MCPAM composite polymer dosage/dye concentration is obtained for low concentration of dye. A dosage as high as $1200 \mathrm{mg} / \mathrm{L}$ is needed to flocculate reactive dye even at a low concentration of dye $(200 \mathrm{mg} / \mathrm{L})$ due to the low probability of molecular contact.

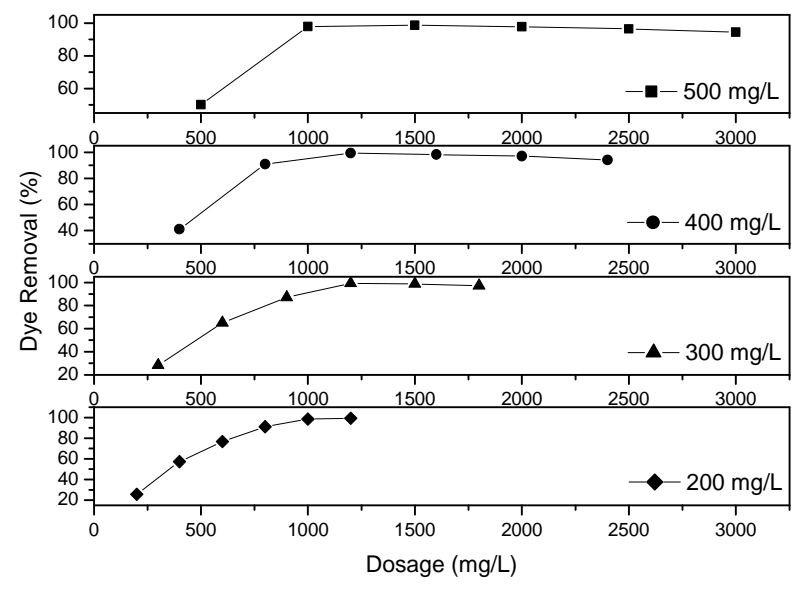

Fig. 5. Effect of dosage of MCPAM composite polymer at different dye concentration.

A qualitative comparison of reactive dye wastewater before and after flocculation is shown in Figure 6 (a) and (b). It is noted in Figure 6 (a) that flocs settled and form sludge at the bottom of the beaker. The filtered supernatant is observed to be transparent in colour. The results show that MCPAM composite polymer performs well in flocculating reactive dye wastewater. 


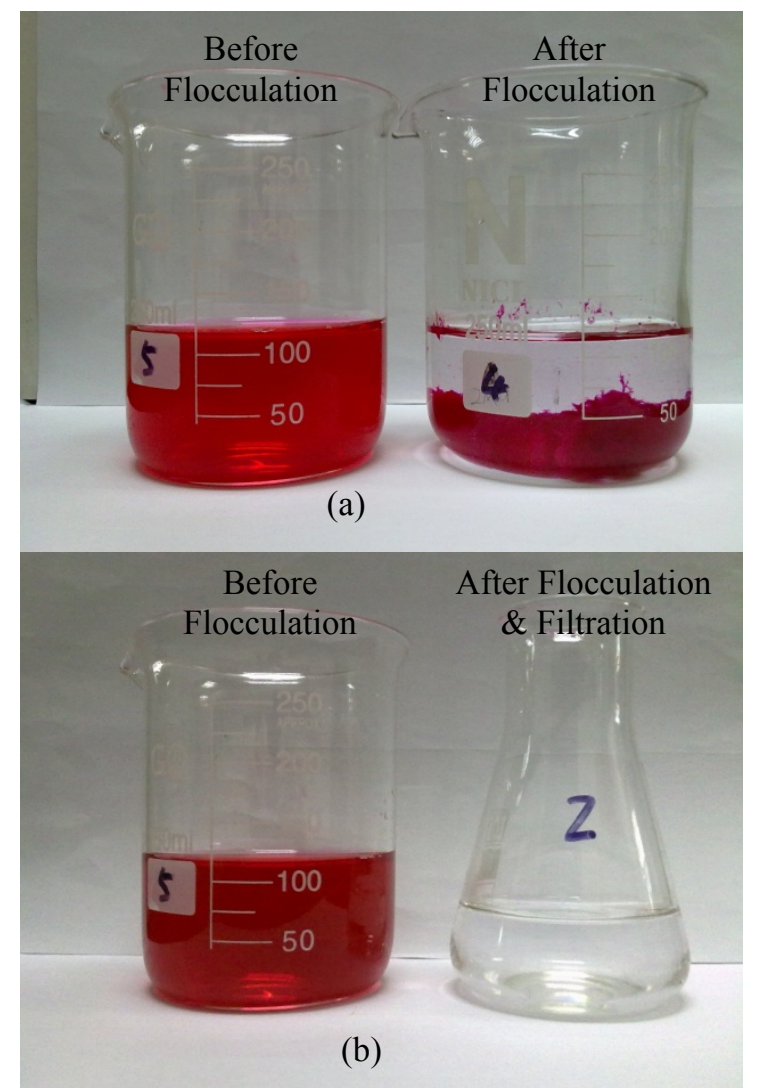

Fig. 6. Comparison of reactive dye wastewater (a) before and after flocculation (b) before flocculation and after flocculation \& filtration.

\section{CONCLUSION}

An inorganic-organic composite polymer (MCPAM) was applied to flocculate simulated reactive dye wastewater. Factors such as $\mathrm{pH}$, concentration of dye, dosage of MCPAM composite polymer and temperature have been taken into account to investigate the dye removal (\%). The result showed that $\mathrm{pH} 12$ was the best working $\mathrm{pH}$ in flocculating dye from wastewater. Temperature showed a remarkable effect on the flocculation of reactive dye where temperature limits the efficiency of dye removal (\%) when temperature increased from 20 to $50^{\circ} \mathrm{C}$. It is recommended that flocculation of reactive dye wastewater to be conducted near room temperature $\left(30^{\circ} \mathrm{C}\right)$. Effect of dosage on the dye removal (\%) was studied with respect to concentration of dye. MCPAM composite showed a good dye removal (\%) where it was able to remove $99 \%$ of dye from all dye concentrations $(200-500 \mathrm{mg} / \mathrm{L})$. However, the dye removal (\%) decreased after it achieved the maximum efficiency. This indicates the re-suspension of destabilized flocs into the supernatant.

\section{ACKNOWLEDGMENT}

The authors gratefully acknowledge financial support from Universiti Sains Malaysia in the form of postgraduate fellowship as well as Research University grant (1001/PTEKIND/814049) which have resulted in this paper.

\section{REFERENCES}

[1] J. Zou, H. Zhu, F. Wang, H. Sui, and J. Fan, "Preparation of a new inorganic-organic composite flocculant used in solid-liquid separation for waste drilling fluid," Chem. Eng. J., vol. 171, pp.350-356, 2011.

[2] K. E. Lee, T. T. Teng, N. Morad, B. T. Poh, and Y. F. Hong, "Flocculation of kaolin in water using novel calcium chloride-polyacrylamide $\left(\mathrm{CaCl}_{2}\right.$-PAM) hybrid polymer," Sep. Purif. Technol., vol. 75, pp. 346-351, 2010.

[3] K. E. Lee, T. T. Teng, N. Morad, B. T. Poh, and M. Mahalingam, "Flocculation activity of novel ferric chloride-polyacrylamide ( $\mathrm{FeCl}_{3}$-PAM) hybrid polymer," Desalination, vol. 266, pp. 108-113, 2011.

[4] J. Addai-Mensah, and C. A.Prestidge, "Structure Formation in Dispersed Systems" in Coagulation and flocculation, $2^{\text {nd }}$ ed. $\mathrm{H}$. Stechemesser, and B. Dobias, Ed. Boca Raton: Taylor \& Francis Group, 2005, pp. 135-216.

[5] M. Natalia, and D. Olli, "Environmental implications of aggregations phenomena: current understanding," Curr. Opin. Colloid In., vol. 11, pp. 246-266, 2006.

[6] P. Somasundaran, V. Runkana, and P.C. Kapur, "Flocculation and Dispersion of Colloidal Suspensions by Polymers and Surfactants: Experimental and Modeling Studies" in Coagulation and flocculation, $2^{\text {nd }}$ ed. H. Stechemesser, and B. Dobias, Ed. Boca Raton: Taylor \& Francis Group, 2005, pp. 767-804.

[7] B. Gao, Q. Yue, and J. Miao, "Evaluation of polyaluminium ferric chloride (PAFC) as a composite coagulant for water and wastewater treatment," Water Sci. Technol., vol. 47, pp. 127-132, 2003.

[8] B. Y. Gao, Y. Wang, and Q. Y. Yue, "The chemical species distribution of aluminum in composite flocculants prepared from polyaluminum chloride (PAC) and polydimethyldiallylammonium chloride (PDMDAAC)," Acta Hydroch. Hydrob., vol. 33, pp. 365-371, 2005.

[9] Z. M. Liu, Y. M. Sang, Z. G. Tong, Q. H. Wang, and T. C. Sun, "Decolourization performance and mechanism of leachate secondary effluent using Poly-aluminium(III)-magnesium(II) sulphate," Water Environ. J., doi: 10.1111/j.1747-6593.2011.00266.x, 2011.

[10] N. D. Tzoupanos, and A. I. Zouboulis, "Preparation, characterisation and application of novel composite coagulants for surface water treatment," Water Res. vol. 45, pp. 3614-3626, 2011.

[11] V. Golob, A. Vinder, and M. Simonic, "Efficiency of the coagulation/flocculation method for the treatment of dyebath effluents," Dyes Pigm., vol. 67, pp. 93-97, 2005.

[12] K. E. Lee, I. Khan, N. Morad, T. T. Teng, and B. T. Poh, "Physicochemical and rheological properties of novel magnesium salt-polyacrylamide composite polymers," J. Dispersion Sci. Technol. 2011. (accepted for publication)

[13] B-Y. Gao, Q-Y. Yue, Y. Wang, and W-Z. Zhou, "Color removal from dye-containing wastewater by magnesium chloride," J. Environ. Manage. vol. 82, pp. 167-172, 2007.

[14] X. Tian, D. Wu, J. Zhang, S. Wang, and X. Jiang, "Flocculated decolorization of vinylsulfone reactive dye solutions with a b-cyclodextrin-based copolymer," J. Appl. Polym. Sci. vol. 118, pp. 480-485, 2010. 